\title{
Simple paper architecture modifications lead to enhanced sensitivity in nanoparticle based lateral flow immunoassay
}

\author{
Claudio Parolo $^{a}$, Mariana Medina-Sánchez ${ }^{a}$, Alfredo de la Escosura-Muñiz ${ }^{a}$, Arben Merkoçi*a,b \\ Received (in $X X X, X X X)$ Xth $X X X X X X X X X 20 X X$, Accepted $X$ th $X X X X X X X X X 20 X X$ \\ ${ }_{5}$ DOI: $10.1039 / b 000000 x$
}

Lateral flow immunoassay (LFIA) are ideal biosensors to detect proteins, but their lack of sensitivity avoids their extensive use. We report a strategy that yields up to an 8-fold improvement in a gold nanoparticles-based LFIA, changing 10 the sizes of the pads. Theoretical flow simulations of the developed LFIA architectures are in concordance with the experimental results.

\section{Introduction}

The sensitive detection of proteins is of tremendous interest in 15 everyday diagnostic, since many of them are biomarkers of diseases. ${ }^{1}$ An early detection of such biomarkers could allow starting a treatment in an early stage of disease, making possible to save many lives. This is particularly important in third world countries, where advanced and expensive technologies are 20 precluded to the foremost of people. ${ }^{2,3}$ The same situation can be found in extreme regions or battlefields, where the conditions do not allow the use of complicate devices and trained personnel cannot be present. For these reasons, it is of extremely importance the development of biosensors, which fulfill the 25 requirements of an ASSURED biosensor ${ }^{4}$ : affordable, sensitive, specific, user-friendly, rapid and robust, equipment free and deliverable to end-users.

Lateral flow immunoassays (LFIA) can be considered as biosensors which fit the definition of ASSURED technology. ${ }_{30}$ Since the first pregnancy test, sold the mid-1970s, the LFIAs have gained much more importance in the field of biosensing. ${ }^{5}$ However, some limitations have avoided their extensive diffusion to other fields, where quantitative analysis together with a better sensitivity are required. In order to answer these demands, the 35 integration of several nanomaterials in paper based biosensors has been already done. ${ }^{6,7}$ Taking advantage of the outstanding properties of materials at nanometer scale, it is possible to improve the performances of the biosensors. ${ }^{8-11}$ Examples of nanomaterials used in lateral flow assays are: gold nanoparticles 40 (AuNPs), ${ }^{12-15}$ quantum dots, ${ }^{16,17}$ carbon nanotubes, ${ }^{18}$ magnetic nanoparticles, ${ }^{19,20}$ and liposomes ${ }^{21,2}$ between others.

In this technical note, we show theoretically and experimentally how simple changes in the architecture of an AuNPs based LFIA, such as width of the sample and conjugation pads, can be 45 transduced to an increased sensitivity and improved detection limit of this analytical device.

\section{Materials and Methods}

\section{Chemicals and equipment}

50 All the materials used for the production of the strips were purchased from Millipore (Billerica MA 08128, USA): sample and absorbent pads (CFSP001700), conjugation pad (GFCP00080000), detection pad (SHF2400425) and backing card (HF000MC100). The membranes were characterized using the ${ }_{55}$ Scanning Electron Microscopy (SEM) (ZEISS MERLIN FESEM). Human IgG whole molecule (HIgG) (I2511), antibody anti-human $\mathrm{IgG}(\alpha \mathrm{HIgG})$ whole molecule (produced in goat; I1886), antibody anti-human IgG $\gamma$ chain specific biotinylated (produced in goat; B1140) and all the chemical reagents 60 (analytical grade) used for the preparation of AuNPs and buffer solutions were purchased from Sigma Aldrich (Spain). Anti-goat IgG ( $\alpha$ GIgG) (produced in chicken; ab86245) was purchased from Abcam (U.K.). The stirrer used was a TS-100 Thermo shaker (BioSan, Latvia). A thermostatic centrifuge Sigma 2-16 ${ }_{65}$ PK (Fisher Bioblock Scientific, France) was used to purify the conjugates of AuNPs/antibodies. An IsoFlow reagent dispensing system (Imagene Technology, USA) was used to dispense the detection and control lines. A strip reader (COZART SpinReact, U.K.) was used for quantitative measurements. A 70 guillotine Dahle 533 (Germany) was used to cut the strips. mQ water, produced using a Milli-Q system (>18.2M $\Omega \mathrm{cm}-1)$ purchased from Millipore (Sweden), was used for the preparation of all the solutions.

The mathematical simulations were done using the module of 75 chemical reaction engineering of the Comsol Multiphysicis 3.4. software, and taking advantage of the equations of transport in porous media.

\section{${ }_{80}$ Preparation and modification of gold nanoparticles}

AuNPs of $20.99 \pm 2.72 \mathrm{~nm}$ in diameter were synthesized according to the citrate reduction of $\mathrm{HAuCl}_{4}$ (as pioneered by Turkevich $^{22}$ ); details of the synthesis can be found in the supplementary information as well as the experimental procedure 85 for the functionalization of AuNPs with $\alpha \mathrm{HIgG} \gamma$ chain specific. The AuNPs modified with the antibody were centrifuged in order to concentrate them 5 times in $2 \mathrm{mM}$ borate buffer $\mathrm{pH} 7.4$ containing $10 \%$ of sucrose. 


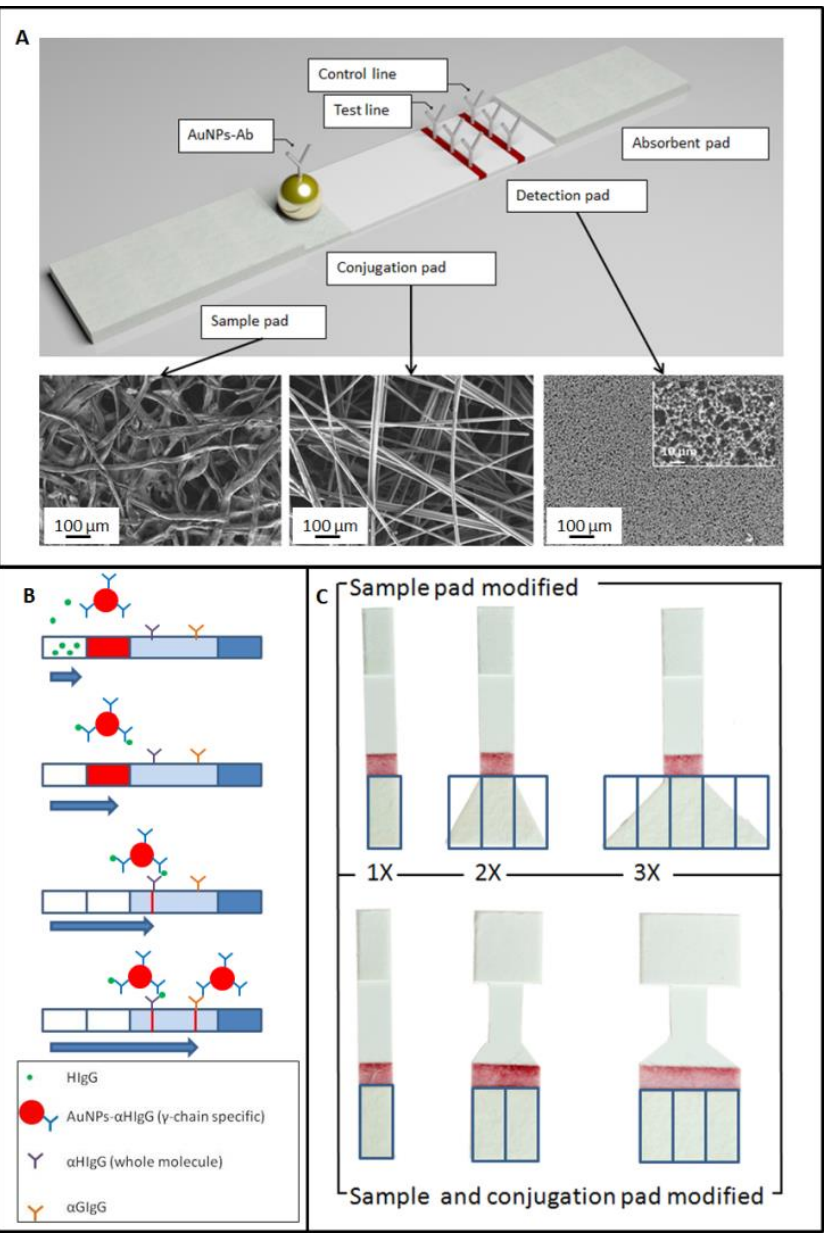

Fig.1 (A) Scheme, not in scale, of a LFIA strip based on a sandwich format (up) and SEM images of the different pads (down). (B) Scheme of

5 the formation of the immune-complex during the flow. (C) Different sets up of the LFIA: with only the sample pad $1 \mathrm{X}, 2 \mathrm{X}$ and $3 \mathrm{X}$ (up) and with both the conjugation and sample pads $1 \mathrm{X}, 2 \mathrm{X}$ and $3 \mathrm{X}$ (down).

\section{Preparation of LFIA strips}

The sample pad was pre-treated by dipping it into $10 \mathrm{mM}$ PBS 10 buffer $\mathrm{pH} 7.4$, containing 5\% BSA and $0.05 \%$ Tween 20 and then dried for $30 \mathrm{~min}$ at $60^{\circ} \mathrm{C}$. The conjugation pad was dipped into the AuNPs solution and then dried for 1 hour under vacuum. The control and the detection lines were obtained dispensing respectively the antibody $\alpha \mathrm{HIgG}$ whole molecule and the $\alpha \mathrm{GIgG}$ 15 onto the detection pad. The antibody solutions at $1 \mathrm{mg} / \mathrm{mL}$ in 10 $\mathrm{mM}$ phosphate buffer $\mathrm{pH} 7.4$ were dispensed with a rate of 1 $\mu \mathrm{L} / \mathrm{cm}$ using the IsoFlow reagent dispensing system. The pad was then dried at $37^{\circ} \mathrm{C}$ for $30 \mathrm{~min}$. After that, the different pads were laminated on the backing card in the following order: first the 20 detection pad, then the absorbent pad at the end of the backing card and overlapping the detection pad, after that the conjugation pad overlapping the detection pad and finally the sample pad on the beginning of the backing card and overlapping the conjugation pad (see figure 1A). All the overlaps were around 1 $25 \mathrm{~mm}$.

After the lamination, the strips were cut using a guillotine to define the external edges and then with a manual cutter to define the internal ones. In order to study the effects of the sample and conjugation pad sizes, two different set-ups were used: changing 30 just the sample pad size and changing both the sample and the conjugation pad sizes. In both cases the surface area studied was $1 \mathrm{X}, 2 \mathrm{X}$ and $3 \mathrm{X}$ the original one.

\section{Assay procedure}

${ }_{35}$ For the $1 \mathrm{X}, 2 \mathrm{X}$ and $3 \mathrm{X}$ strip sizes, the assays were performed pipetting at the bottom of the sample pad 200, 400 and $600 \mu \mathrm{L}$ of the HIgG solution respectively. A blank and three different concentrations of $\mathrm{HIgG}$ were studied: 6,60 and $600 \mathrm{ng} / \mathrm{mL}$ in 10 $\mathrm{mM}$ PBS $\mathrm{pH}$ 7.4. The assay took around $10 \mathrm{~min}$ to develop the 40 color of the lines and $10 \mathrm{~min}$ for the washing step, performed pipetting the same amount of buffer. The strip was finally cut with a uniform wide of $8 \mathrm{~mm}$ in order to be read by the strip reader.

The assay followed an immunosandwich format: the $\alpha \mathrm{HIgG}(\gamma-$ ${ }_{45}$ chain specific) antibodies, attached to the AuNPs, recognized the $\gamma$-chain of the HIgG of the sample. The conjugates AuNPs$\alpha \mathrm{HIgG}(\gamma$-chain specific)-HIgG are further stopped at the detection line by the $\alpha \mathrm{HIgG}$ (whole molecule) antibodies fixed at the detection line. Stronger is the red color of the AuNPs at the 50 detection line and higher is the concentration of the analyte (HIgG) present in the sample (figure 1B). Furthermore the $\alpha \mathrm{GIgG}$ of the control line recognized the $\alpha \mathrm{HIgG}$ ( $\gamma$-chain specific) of the complexes which are not stopped at the detection line, confirming that the assay worked properly.

55

\section{Mathematical simulations}

The flow in the strip membranes is usually described by the Navier-Stokes equation (pores-free region), and using the Brinkman equations (porous region). The most common way to ${ }_{60}$ deal with pores-free and porous media flow in a system is to couple Darcy's law, which does not account for viscous effects, with the Navier-Stokes equations. However, depending on the pore size distribution of the porous media and the fluid's properties, it is not always appropriate to neglect viscous effects.

65 The Brinkman equations account for momentum transport through viscous effects and through pressure gradients in porous media, and can be considered an extension of Darcy's Law, which is a derived constitutive equation that describes the flow of a fluid through a porous medium (see equation 1$)^{23}$ :

70

$Q=\frac{k A}{\mu}\left(\frac{d P}{d L}\right)$

where $\mathrm{Q}$ is the flow rate (in units of volume for time unit), $\mathrm{k}$ is the relative permeability (typically in millidarcies), $\mathrm{A}$ is the cross sectional area (in square meters), $\mu$ is the viscosity of the fluid (in 75 centipoises), L the length of the porous media (in meters) and $\mathrm{dP} / \mathrm{dL}$ is the pressure change per unit length. The constants for the different pads were calculated empirically measuring the volume of water absorbed by each pad. In this way it was possible to estimate the porosity and the permeability of the ${ }_{80}$ different pads (see supplementary information).

The initial conditions defined for the simulation were: porosity and permeability of the membranes (empirically calculated, as 
detailed at the supplementary information) and viscosity and density of the fluid (water). On the other hand, for the boundary conditions the initial velocity was calculated from the volume of the liquid introduced in the membrane $(200,400$, and $600 \mu \mathrm{L}$ for ${ }_{5}$ the $1 \mathrm{X}, 2 \mathrm{X}$ and $3 \mathrm{X}$ strip respectively), the cross area and the time necessary to absorb the respective volume. So for $1 \mathrm{X}, 2 \mathrm{X}$ and $3 \mathrm{X}$ strip the velocity was $1.47 \times 10^{-3} \mathrm{~m} / \mathrm{s}, 2.94 \times 10^{-3} \mathrm{~m} / \mathrm{s}$ and $4.41 \mathrm{x}$ $10^{-3} \mathrm{~m} / \mathrm{s}$ respectively.

\section{${ }_{10}$ Results and Discussion}

\section{Effect of the architecture of the sample pad}

A scheme of the LFIA performed for the detection of $\mathrm{HIgG}$ as model analyte is shown in figure 1B. It was first evaluated how the changes in only the sample pad size could affect the 15 sensitivity of the LFIA. The sample pads were designed to have a trapezoid shape to facilitate the flow. In order to obtain the sample pad area 2 and 3 times bigger, the smaller base of the trapezoid, the one in contact with the conjugation pad, was fixed at $8 \mathrm{~mm}$ wide, whereas the bigger base was increased 20 respectively to $24 \mathrm{~mm}$ and $40 \mathrm{~mm}$, as detailed in figure $1 \mathrm{C}$. The strip reader gives as output \% values corresponding to the intensity of the lines: weaker is the intensity of the line and higher is the $\%$ value. The blank of each strip was subtracted to the results obtained to compensate possible non-specific interactions.

${ }_{25}$ In figure $2 \mathrm{~A}$ it is possible to see how the strips look like after an assay with $60 \mathrm{ng} / \mathrm{mL}$ of $\mathrm{HIgG}$. There are not clear differences in the sensitivity for the different geometries used. In fact as schematized in figure $2 \mathrm{~B}$ there are two opposite effects that influenced the assay: the amount of analyte and the speed of the 30 flow. Using bigger sample pads it is possible to use more volume of the sample, and consequently more analyte is available, but this induces an increment in the speed of the flow, which reduces the time that the AuNPs labels have to bind the analyte. The flow is also faster in the detection pad, decreasing the time to 35 recognize the immune-complexes formed by the antibodies of the test and control lines. As represented by the arrows in the figure, in the $1 \mathrm{X}$ format the AuNP speed is low giving enough time to have a good recognition of the analyte, but the analyte amount is not high. The $2 \mathrm{X}$ format has a flow speed higher than the $1 \mathrm{X}$ but 40 still not enough fast to compromise the interaction between the antibodies and the analyte; furthermore the amount of analyte is bigger, increasing the possibility of recognition by the AuNPs. Finally, in the $3 \mathrm{X}$ format the speed is very fast giving not enough time to the AuNP complexes to interact with the antigen, 45 consequently decreasing the limit of detection of the assay even if the amount of analyte is bigger.

These phenomena were also evaluated by mathematical simulations (see figure 2C). For each geometry the flow speed was calculated at the level of the conjugation pad and the test ${ }_{50}$ line. For $1 \mathrm{X}$ geometry the speeds resulted to be respectively $2.1 \mathrm{x}$ $10^{-3}$ and $1.95 \times 10^{-3} \mathrm{~m} / \mathrm{s}$, whereas for the $2 \mathrm{X}$ they were $8 \times 10^{-3}$ and $7.2 \times 10^{-3} \mathrm{~m} / \mathrm{s}$ and finally $18 \times 10^{-3}$ and $16 \times 10^{-3} \mathrm{~m} / \mathrm{s}$ for the $3 X$.

The graph in figure 2D shows the results obtained with the strip 55 reader. They are in accordance with the theoretical calculations. In fact, there is not any clear positive effect in the LFIA sensitivity changing only the sample pad size. For the strips with the sample pad 2 times bigger it is possible to observe a slight increased sensitivity of the assay, probably due to the higher 60 volume of sample, which implies a higher amount of analyte. However, the results obtained using the strips with the sample pad 3 times bigger show that the sensitivity of the assay is lower than the one obtained with the sample pad of the original size. This can be explained considering that in the $3 \mathrm{X}$ configuration 65 the flow has a speed of approximately one order of magnitude higher than the 1X. Furthermore the AuNPs are re-suspended by a fixed amount of liquid and they are drugged by it. This means that just the analyte present in such volume of liquid can be recognized by the AuNP labels, making not useful using an 70 excess of volume.

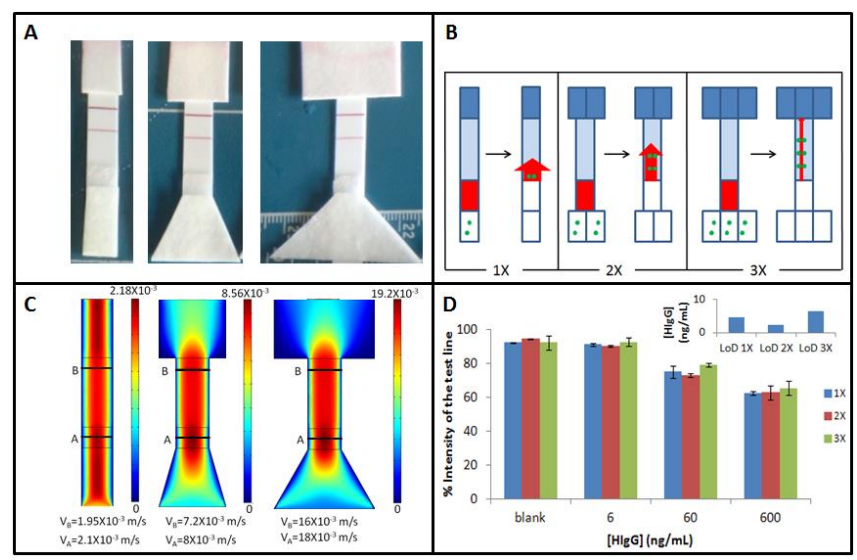

Fig. 2 (A) Photos of LFIA with different sample pad architectures for 60 $\mathrm{ng} / \mathrm{mL}$ HIgG. (B) Scheme of the two opposite effects: the amount of 75 analyte vs the speed of the flow for the LFIAs using a bigger sample pad. The red arrows represent the speed of the AuNPs in the flow and the green point stated for the analyte. (C) Results of the flow speed simulations for sample pads with different sizes. (D) Effect of the sample pad relative size on the quantitative measurement for different $\mathrm{HIgG}$ 80 concentrations and the corresponding LODs obtained (inset).

\section{Effect of different architectures for both sample and conjugation pad.}

In the second study, both the conjugation and the sample pads 85 were changed. Here, the shapes of the sample and conjugation pads remain rectangular, so they are simply made 2 and 3 times bigger (figure 1C). In figure $3 \mathrm{~A}$ it is possible to observe how the strips look like after an assay for $60 \mathrm{ng} / \mathrm{mL}$ of $\mathrm{HIgG}$. It is evident an increase in the intensity of the band increasing the sizes of 90 sample and conjugation pads. In fact, as schematized in figure $3 \mathrm{~B}$, using these configurations the flow speed in the conjugation pad should not change significantly, giving enough time to the AuNPs labels to recognize the analyte. In addition, using a bigger volume there were more analyte molecules to be detected and at 95 the same time, since also the conjugation pad is bigger, more AuNPs to be used as labels. This brought out the formation of a higher number of immuno-complexes. Just when the flow passed to the detection pad, there was an increment in the flow speed, but this phenomenon was compensated by the bigger number of 100 labels, which recognized the analyte. The theory was confirmed by the mathematical simulations (figure $3 \mathrm{C}$ ): for the conjugation 
pad the speed values were of the same order: $2.1 \times 10^{-3}, 4.4 \times 10^{-}$ ${ }^{3}$ and $4.4 \times 10^{-3} \mathrm{~m} / \mathrm{s}$ for $1 \mathrm{X}, 2 \mathrm{X}$ and $3 \mathrm{X}$ respectively. However for the detection pad a high increase in the value is obtained when increasing the size, being $1.95 \times 10^{-3}, 7.8 \times 10^{-3}$ and $17.8 \times 10^{-3}$ $5 \mathrm{~m} / \mathrm{s}$ respectively. The results obtained with the strip reader (figure 3D) confirmed all the previous data, showing limits of detection for the strips $1 \mathrm{X}, 2 \mathrm{X}$ and $3 \mathrm{X}$ to be respectively: $5.89,1.83$ and 0.7 $\mathrm{ng} / \mathrm{mL}$. This means that increasing 3 times the width of the conjugation and sample pads it is possible to obtain an 8 -fold 10 improvement in the limit of quantification.

These results could be further improved by increasing the difference between the width of the detection pad and those of the conjugation and sample pads. This can be achieved making wider the conjugation and sample pad and/or making smaller the 15 detection pad. However, some drawbacks are previewed: with bigger conjugation and sample pads, more volume of the sample as well as more amount of label is required. On the other hand, smaller detection pads would not be compatible with the strip reader. Another point to be considered is the shape of the strips:

20 longer strips would allow a softer pre-concentration, which would probably produce more reproducible results. This can also be achieved placing the detection line closer to the end of the detection pad.

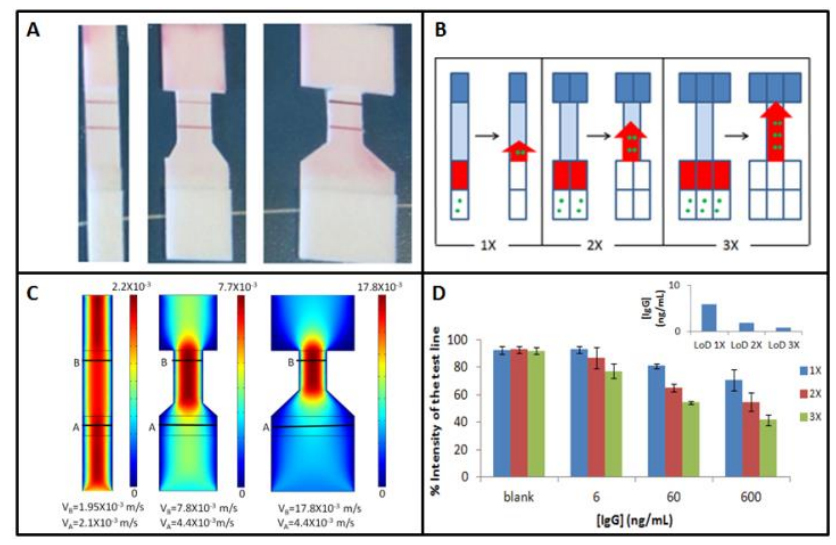

${ }_{25}$ Fig. 3 (A) Photos of LFIA with different sample and conjugation pads architectures for $60 \mathrm{ng} / \mathrm{mL} \mathrm{HIgG.} \mathrm{(B)} \mathrm{Scheme} \mathrm{of} \mathrm{the} \mathrm{two} \mathrm{opposite} \mathrm{effects:}$ the amount of analyte $v s$ the speed of the flow for the LFIAs using bigger sample and conjugation pads. The red arrows represent the speed of the AuNPs in the flow and the green point stated for the analyte. (C) Results 30 of the flow speed simulations for sample and conjugation pads with different sizes. (D) Effect of the sample and conjugation pads relative sizes on the quantitative measurement for different $\mathrm{HIgG}$ concentrations and the corresponding LODs obtained (inset).

\section{${ }_{35}$ Conclusions}

We have demonstrated that very simple changes of the LFIA architecture, like increasing the size of both the conjugation and the sample pads, can improve its performance in terms of sensitivity of the assay. Flow speed simulations also corroborate 40 the experimental achievements and can represent useful tools in designing novel lateral flow arquitectures. The proposed designs can be easily applied in any type of LF strips without changing their fabrication method; moreover it is simple and cheap, fostering its use for point of care applications, even at the doctor
45 office or in undeveloped countries. Studies using a different method of detection, based on the use of a camera, and the use of a wax printer, in order to define better the shape of the LFIA, have already started in our lab and they will enable decreasing the size of the detection pad enabling a further increment in the 50 sensitivity of the device.

\section{Acknowledgments}

We acknowledge MEC (Madrid) for the project MAT201125870, the E.U.'s support under FP7 contract number 246513 ${ }_{55}$ "NADINE" and the NATO Science for Peace and Security Programme's support under the project SfP 983807.

\section{Notes and references}

${ }^{a}$ Nanobioelectronics \& Biosensors Group, Institut Català de 60 Nanotecnologia, CIN2 (ICN-CSIC), Campus UAB, Barcelona, Spain

${ }^{b}$ ICREA, Barcelona, Spain E-mail: arben.merkoci@icn.cat

$\dagger$ Electronic Supplementary Information (ESI) available: [details of any supplementary information available should be included here]. See DOI: $10.1039 / \mathrm{b} 000000 \mathrm{x}$

65 1. D. Mabey, R. W. Peeling, A. Ustianowski, and M. D. Perkins, Nature reviews. Microbiology, 2004, 2, 231-40.

2. P. Yager, T. Edwards, E. Fu, K. Helton, K. Nelson, M. R. Tam, and B. H. Weigl, Nature, 2006, 442, 412-8.

3. A. K. Ellerbee, S. T. Phillips, A. C. Siegel, K. a Mirica, A. W. 70 Martinez, P. Striehl, N. Jain, M. Prentiss, and G. M. Whitesides, Analytical chemistry, 2009, 81, 8447-52.

4. R. W. Peeling, K. K. Holmes, D. Mabey, and a Ronald, Sexually transmitted infections, 2006, 82 Suppl 5, v1-6.

5. G. a Posthuma-Trumpie, J. Korf, and A. van Amerongen, 75 Analytical and bioanalytical chemistry, 2009, 393, 569-82.

6. S. Song, Y. Qin, Y. He, Q. Huang, C. Fan, and H.-Y. Chen, Chemical Society reviews, 2010, 39, 4234-43.

7. C. Parolo and A. Merkoçi, Chemical Society Reviews, 2013. DOI: $10.1039 / \mathrm{C} 2 \mathrm{CS} 35255 \mathrm{~A}$

808 G. Aragay, J. Pons, and A. Merkoçi, Chemical reviews, 2011, 111, 3433-58.

9. M. Perfézou, A. Turner, and A. Merkoçi, Chemical Society reviews, 2011, 41, 2606-2622.

10. A. de la Escosura-Muñiz, C. Parolo, and A. Merkoçi, Materials 85 Today, 2010, 13, 24-34.

11. M. Medina-Sánchez, S. Miserere, and A. Merkoçi, Lab on a Chip, 2012, 12, 2000-2005.

12. Y. He, S. Zhang, X. Zhang, M. Baloda, A. S. Gurung, H. Xu, X. Zhang, and G. Liu, Biosensors \& bioelectronics, 2011, 26, 90 2018-24. 
13. S. Lou, J.-Y. Ye, K.-Q. Li, and A. Wu, The Analyst, 2011, 137, 1174-1181.

14. W. Zhao, M. M. Ali, S. D. Aguirre, M. A. Brook, and Y. Li, Analytical chemistry, 2008, 80, 8431-8437.

5 15. C. Parolo, A. de la Escosura-Muñiz, and A. Merkoçi, Biosensors and Bioelectronics, 2012, DOI: 10.1016/j.bios.2012.06.049.

16. X. Zhu, L. Chen, P. Shen, J. Jia, D. Zhang, and L. Yang, Journal of agricultural and food chemistry, 2011, 59, 2184-9.

$1017 . \quad$ Z. Zou, D. Du, J. Wang, J. N. Smith, C. Timchalk, Y. Li, and Y. Lin, Analytical chemistry, 2010, 82, 5125-33.

18. A. Abera and J.-W. Choi, Analytical Methods, 2010, 2, 18191822.

19. S. Puertas, M. Moros, R. Fernández-Pacheco, M. R. Ibarra, V. 15 Grazú, and J. M. de la Fuente, Journal of Physics D: Applied Physics, 2010, 43, 474012.

20. D. Tang, J. C. Sauceda, Z. Lin, S. Ott, E. Basova, I. Goryacheva, S. Biselli, J. Lin, R. Niessner, and D. Knopp, Biosensors \& bioelectronics, 2009, 25, 514-8.

2021 S. Shukla, H. Leem, and M. Kim, Analytical and bioanalytical chemistry, 2011, 401, 2581-90.

22. J. Turkevich, J., Stevenson, P., Hillier, Discuss. Faraday Soc., 1951, 55-75.

23. S. Chen and G. D. Doolen, Annu. Rev. Fluid Mech., 1998, 30,

25 329-64. 


\section{Supplementary information}

Simple paper architecture modifications lead to enhanced sensitivity in nanoparticle based lateral flow immunoassay

${ }_{5}$ Claudio Parolo ${ }^{1}$, Mariana Medina-Sáncheza ${ }^{1}$, Alfredo de la Escosura-Muñiz ${ }^{1}$, Arben Merkoçi ${ }^{1,2 *}$

${ }^{1}$ Nanobioelectronics \& Biosensors Group, Institut Català de Nanotecnologia, CIN2 (ICN-CSIC), Campus UAB, Barcelona, Spain

${ }^{2}$ ICREA, Barcelona, Spain

*Corresponding author: arben.merkoci@icn.cat Tel: +34935868014; fax: +34935813797 


\section{Preparation of gold nanoparticles (AuNPs)}

All glassware used in this preparation was thoroughly cleaned in aqua regia overnight and rinsed with double distilled $\mathrm{H}_{2} \mathrm{O}$ and reflux was used for all the procedure which was done as follows: a $50 \mathrm{~mL}$ ${ }_{5}$ aqueous solution of $0.01 \% \mathrm{HAuCl}_{4}$ was heated to boiling and vigorously stirred in a $250 \mathrm{~mL}$ roundbottom flask; $5 \mathrm{~mL}$ of $40 \mathrm{mM}$ sodium citrate were added quickly to this solution. Boiling was continued for an additional $10 \mathrm{~min}$. The solution was cooled to room temperature with a continuous stirring for another $15 \mathrm{~min}$. The colloids were stored in dark bottles at $4^{\circ} \mathrm{C}$.

\section{${ }_{10}$ AuNPs modification with antibodies}

The AuNPs were then conjugated with the antibody $\alpha \mathrm{HIgG} \gamma$ chain specific. Briefly, $1.5 \mathrm{ml}$ of AuNPs solution was adjusted to $\mathrm{pH} 9$ with $10 \mathrm{mM}$ borate buffer $\mathrm{pH} 9.2$. Then, without stopping the stirring, $100 \mu \mathrm{L}$ of the antibody solution $(100 \mu \mathrm{g} / \mathrm{mL})$ were added drop by drop and the resulting solution was ${ }_{15}$ incubated for $20 \mathrm{~min}$ at $650 \mathrm{rpm}$. Then $100 \mu \mathrm{L}$ of $1 \mathrm{mg} / \mathrm{mL}$ BSA aqueous solution were added drop by drop and the stirring was continued for other $20 \mathrm{~min}$ at $650 \mathrm{rpm}$. Finally the solution was centrifuged at $14000 \mathrm{rpm}$ for $20 \mathrm{~min}$. The supernatant was removed and the pellet of AuNPs was re-suspended in $300 \mu \mathrm{L}$ of $2 \mathrm{mM}$ borate buffer $\mathrm{pH} 7.4$ containing $10 \%$ of sucrose.

\section{${ }_{20}$ Empirical calculation of pad constants}

The porosity and permeability constants of sample, conjugation and detection pads were calculated measuring the change in a fixed volume of PBS (PBS density $=1,97 \mathrm{~g} / \mathrm{mL}$ ) after dipping the different pads as shown in figure S1. The sizes of the pads are also shown in figure S1. The porosities of the ${ }_{25}$ absorbent, conjugation and detection pad were respectively: $0.47,0.23,0.31$; whereas the permeability 
were: $7.5 \times 10^{-6}, 1.85 \times 10^{-6}, 4.91 \times 10^{-7} \mathrm{~m}^{2}$, calculated considering the transversal area of the membrane and its porosity.

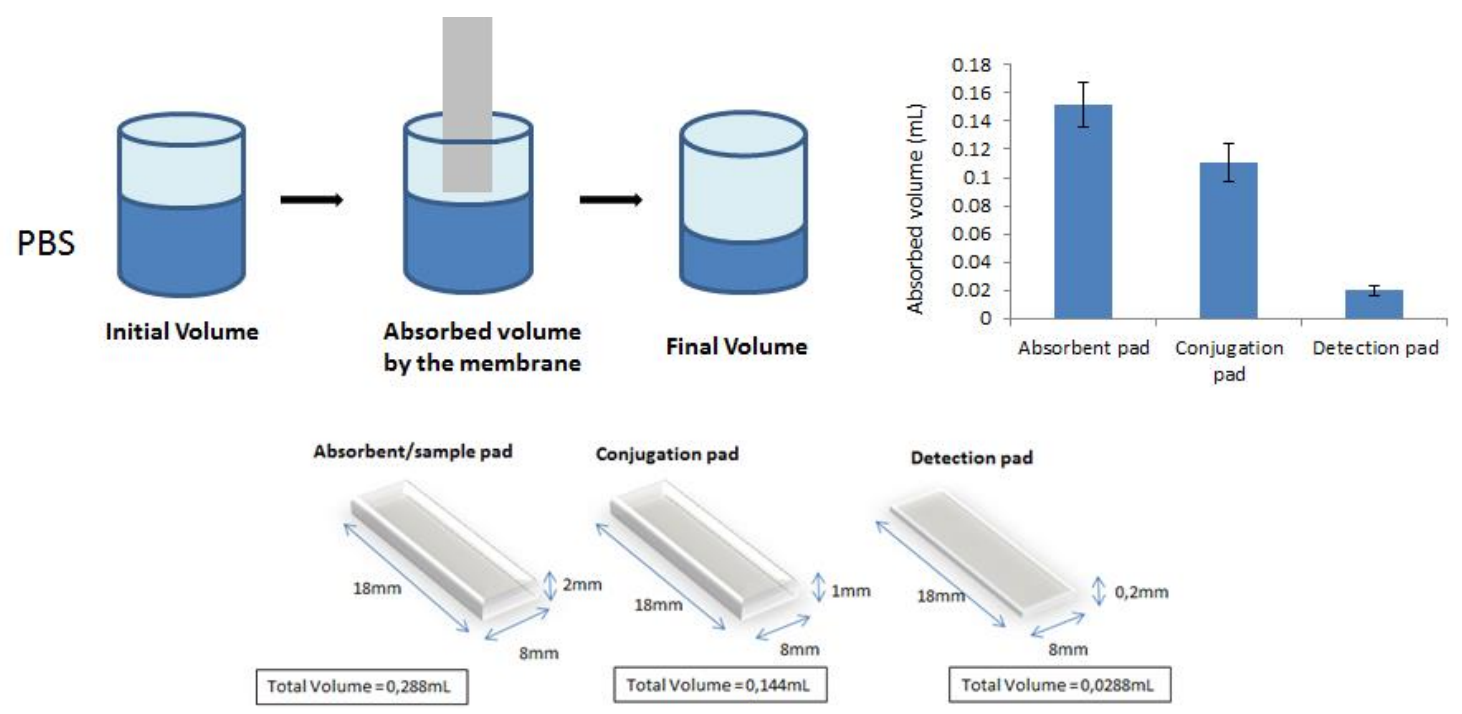

${ }_{5}$ Figure S1: On the left: scheme of the experiment used to calculate the porosity and permeability of the membranes. On the right: results obtained for the different membranes. On the bottom: sizes and total volumes of the membranes used. 